九州大学学術情報リポジトリ

Kyushu University Institutional Repository

Problems in Size Expansion Analysis based on Hypothetical Relationships between Scale Factor, Exponential Function and Bondi K-Factor

Shimojo, Masataka

Laboratory of Regulation in Metabolism and Behavior, Division of Animal and Marine Bioresource

Sciences, Department of Bioresource Sciences, Faculty of Agriculture, Kyushu University

https://doi.org/10.5109/1526401

出版情報：九州大学大学院農学研究院紀要. 60 (2)，pp. 399-403，2015-09-18. Faculty of Agriculture, Kyushu University

バージョン :

権利関係 : 


\title{
Problems in Size Expansion Analysis based on Hypothetical Relationships between Scale Factor, Exponential Function and Bondi K-Factor
}

\author{
Masataka SHIMOJO* \\ Laboratory of Regulation in Metabolism and Behavior, Division of Animal and Marine Bioresource Sciences, \\ Department of Bioresource Sciences, Faculty of Agriculture, Kyushu University, \\ Fukuoka 812-8581, Japan \\ (Received May 11, 2015 and accepted May 19, 2015)
}

\begin{abstract}
Exponential function is used not only for the basic growth analysis of the individual plant or animal in animal agriculture but also for the space expansion analysis in cosmology. This study was designed to investigate the problems in the size expansion analysis of the toy space model based on the hypothetical relationships between scale factor, exponential function and Bondi K-factor. Problems (A) (E) were shown. (A) The toy space model using Bondi K-factor was related to the Minkowski space (flat space-time), which was different from the standard model of cosmic expansion based on the FLRW metric. (B) The toy space model was related to the relativistic Doppler redshift, but not to the cosmological redshift that was used in the standard model. (C) Even if Bondi K-factor was hypothetically and forcedly related to the cosmic scale factor through exponential function, the relativistic Doppler redshift merely gave a very poor approximation to the cosmological redshift. (D) The rapid but decelerated expansion at the early stage of the toy space model was not consistent with the accelerated expansion of the inflationary theory. (E) The toy space model did not give the information on the energy distribution of the universe (baryonic matter, dark matter and dark energy), though this toy model gave a mathematical curve composed of early rapid expansion in decelerated expansion, inflection point and accelerated expansion. In conclusion, this study showed that the toy space model using Bondi $\mathrm{K}$-factor had various problems when compared with the standard model of cosmic expansion.
\end{abstract}

Key words: Bondi K-factor, cosmological redshift, exponential function, relativistic Doppler redshift, scale factor

\section{INTRODUCTION}

Animal agriculture is based on the production of plants and animals. The basic growth analysis of the individual plant (Blackman, 1919) or animal (Brody, 1945) is conducted using exponential function. Exponential function is used also for the description of the accelerated expansion at the early inflationary stage (Starobinsky, 1980; Kazanas, 1980; Sato, 1981; Guth, 1981; Linde, 1982; Albrecht and Steinhardt, 1982) and at the present stage (Riess et al., 1998; Perlmutter et al., 1999) in the cosmic history. Tegmark (2014) describes that the inflation theory says that our baby universe grew much like a human baby. Thus, analyzing the growth of individual animal seems to be mathematically similar to analyzing the cosmic expansion. These are why I am interested in the size expansion analysis using exponential function.

Exponential function is known to be equivalent to Bondi K-factor (for example, Lavenda, 2000), the factor that was proposed by Bondi (1964) as a useful educational tool of the special relativity. Bondi K-factor, through the recession velocity of the moving object, is related to the relativistic Doppler redshift. However, as Weinberg (2008) describes, the standard model of cosmic expansion uses the cosmological redshift that depends on the increase of the cosmic scale factor in the whole

* E-mail: mshimojo@agr.kyushu-u.ac.jp

This study was conducted mostly at Agri-Bio Research Laboratory on Ito Campus, Kyushu University, Motooka, Fukuoka 819-0395 period from the emission to the absorption of light. In recent 15 years, there are many studies on whether or not or how the relativistic Doppler redshift is related to the cosmic history (for example, Bedran, 2002; Macleod, 2004; Chodorowski, 2006; Sitnikov, 2006; Francis et al., 2007; Peacock, 2008; Bunn and Hogg, 2009; Rabounski, 2009; Farley, 2010; Davis, 2010; Ben-Amots, 2011; Rebhan, 2012; Kaiser, 2013; May and Yu, 2013; Wagh, 2013; Kipreos, 2014; Konushko, 2014; Li, 2014; Pierseaux, 2014; MacCallum, 2015; Santilli, 2015). Subsequent reports (Shimojo, 2014, 2015) suggested a hypothetical interpretation of Bondi $\mathrm{K}$-factor as a toy space model, showing a mathematical curve that is composed of early rapid expansion, decelerated expansion, inflection point, and accelerated expansion. However, the standard theory uses the cosmological redshift for the investigation of cosmic expansion.

The present study was designed to investigate the problems in the size expansion analysis of the toy space model based on the hypothetical relationships between scale factor, exponential function and Bondi K-factor.

\section{PROBLEMS IN A TOY SPACE MODEL USING BONDI K-FACTOR}

\section{The standard model of cosmic expansion}

If based on the cosmological principle, then the standard model of cosmic expansion is given by expressions $(1) \sim(5)$, 


$$
\begin{aligned}
& \left(\frac{1}{a} \cdot \frac{d a}{d t}\right)^{2} \\
& =\frac{8 \pi G}{3 c^{2}} \rho-\frac{c^{2} K}{a^{2}}+\frac{c^{2} \Lambda}{3}, \\
& H=\frac{1}{a} \cdot \frac{d a}{d t}, \\
& a \propto \exp (H \cdot t), \\
& \frac{a\left(t_{2}\right)}{a\left(t_{1}\right)}=\exp \left[\int_{t_{1}}^{t_{2}} H d t\right], \\
& Z_{\mathbf{c}}=\frac{a\left(t_{2}\right)}{a\left(t_{1}\right)}-1=\frac{\lambda_{2}}{\lambda_{1}}-1,
\end{aligned}
$$

where $t=$ time, $a=$ scale factor, $\pi=$ circular constant, $G=$ gravitational constant, $c=$ speed of light in vacuum, $\rho=$ energy density, $p=$ pressure, $K=$ curvature of space, $\Lambda=$ cosmological constant, $H=$ Hubble parameter (cosmic expansion rate), $Z_{\mathbf{c}}=$ cosmological redshift from $t_{\mathbf{1}}$ (emmision of light) to $t_{2}$ (absorption of light), $\lambda=$ wavelength.

\section{A toy space model using Bondi K-factor}

A toy space model using Bondi $\mathrm{K}$-factor $[B(v)]$ is given by expressions $(6) \sim(8)$ that are altered from the previous reports (Shimojo, 2014, 2015),

$$
\begin{aligned}
& B(v)=\sqrt{\frac{c-v}{c+v}}, \\
& Z_{\mathbf{R}}=\frac{1}{B(v)}-1=\sqrt{\frac{c+v}{c-v}}-1=\frac{\lambda_{2}}{\lambda_{1}}-1, \\
& B(v) \cdot\left(Z_{\mathbf{R}}+1\right)=B(v) \cdot \frac{1}{B(v)}=1,
\end{aligned}
$$

where $B(v)$ = space size ratio (toy space model), $c=$ speed of light in vacuum, $v=$ recession speed of moving object, $c \geq v \geq-c, 0 \leq B(v)<\infty, Z_{\mathbf{R}}=$ relativistic Doppler redshift, $\lambda=$ wavelength.

\section{Equivalence of Bondi $\mathrm{K}$-factor and exponential function}

Expressions (9) and (10) give expressions (11) and (12) that show the equivalence of Bondi $\mathrm{K}$-factor and exponential function,

$$
\begin{aligned}
& \frac{1}{\sqrt{1-v^{2} / c^{2}}}=\frac{\exp (\theta)+\exp (-\theta)}{2}, \\
& \frac{v / c}{\sqrt{1-v^{2} / c^{2}}}=\frac{\exp (\theta)-\exp (-\theta)}{2},
\end{aligned}
$$

$$
\begin{aligned}
& \sqrt{\frac{c \pm v}{c \mp v}}=\exp ( \pm \theta), \\
& \pm \frac{v}{c}=\frac{\exp ( \pm 2 \theta)-1}{\exp ( \pm 2 \theta)+1},
\end{aligned}
$$

where double-sign corresponds.

\section{Relativistic Doppler redshift as a very poor approx- imation to cosmological redshift}

Expression (3) shows that the cosmic scale factor is proportional to exponential function with a variable of Hubble parameter. Expression (11) shows that Bondi $\mathrm{K}$-factor is described using exponential function. Since the relativistic Doppler redshift $\left(Z_{\mathbf{R}}\right)$ is conceptually different from the cosmological redshift $\left(Z_{\mathbf{c}}\right)$, hypothetically and forcedly relating expressions (7) to (5) through exponential function shows that $Z_{\mathbf{R}}$ merely gives a very poor approximation to $Z_{\mathbf{C}}[(13) \sim(15)]$,

$$
\begin{aligned}
& \sqrt{\frac{c+v}{c-v}} \sim \frac{a\left(t_{2}\right)}{a\left(t_{1}\right)}, \\
& Z_{\mathbf{R}} \sim Z_{\mathbf{C}}, \\
& \frac{v}{c} \sim \frac{\left[a\left(t_{2}\right) / a\left(t_{1}\right)\right]^{2}-1}{\left[a\left(t_{2}\right) / a\left(t_{1}\right)\right]^{2}+1} .
\end{aligned}
$$

There is a hypothesis that $Z_{\mathbf{C}}$ is interpreted as the accumulation of the infinitesimal $Z_{\mathbf{R}}$ (for example, Bunn and Hogg, 2009; Matsubara, 2013). There are some studies that suggest the use of the relativistic Doppler redshift (for example, Bunn and Hogg, 2009; May and Yu, 2013; Pierseaux, 2014). These may give the reason why Bondi $\mathrm{K}$-factor is hypothetically interpreted as a toy space model (Shimojo, 2014, 2015). However, this toy space model is based on the concept that is different from that of the standard model.

\section{Hypothetical phenomena in the size expansion analysis of the toy space model}

Despite the serious problems, the toy space model using Bondi K-factor [(6) (7)] seems to suggest mathematical phenomena (i) (iv) about the space expansion. (i) The toy space model (6) shows an early rapid expansion in the context of the decelerated expansion that continues until the inflection point, at which point there turns into the accelerated expansion. (ii) The inflection point of $B(v)$ is the halfway point $[v=c / 2$, $\left.B(c / 2)=0.577, Z_{\mathbf{R}}=0.732\right]$ between the birth point $[v=$ $\left.c, B(c)=0, Z_{\mathbf{R}}=\infty\right]$ and the present point $[v=0, B(0)=$ $\left.1, Z_{\mathbf{R}}=0\right]$. (iii) The relativistic expansion of the toy space model reflects the balance between the attractive force (baryonic matter and dark matter) and the repulsive force (dark energy). (iv) The relativistic Doppler redshift $\left(Z_{\mathbf{R}}\right)+1$ is related to the energy density of the toy space model. 
Perlmutter et al. (1999) showed that the crossover between the deceleration and the acceleration occurred when redshift $\approx 0.73$. Perlmutter (2011) showed that the best fit curve was decelerating for about the first seven billion years, and then accelerating for the most recent approximately seven billion years. Choi (2010) reported the decelerating expansion until about 7 billion years, and accelerating expansion from 7 billion years ago. Barrow (2011) described that the deceleration was switched to the acceleration when the universe was expanded to about $57 \%$ of the present size.

However, the early decelerated expansion of the toy space model is not consistent with the early accelerated expansion in the cosmic inflation theory proposed by preceding studies (Starobinsky, 1980; Kazanas, 1980; Sato, 1981; Guth, 1981; Linde, 1982; Albrecht and Steinhardt, 1982). In addition, the toy space model does not give the information on the energy distribution of the universe that was reported by preceding studies (Riess et al., 1998; Perlmutter et al., 1999; Komatsu et al., 2009).

The universe is considered almost flat (Guth, 1981; Linde, 1982). Bondi K-factor (relativistic Doppler redshift) is related to the Minkowski space that is flat spacetime. However, the standard model of cosmic expansion is based on the FLRW metric (the cosmological redshift).

\section{Hypothetical relationships between some natural phenomena}

The natural logarithm of expression (8) gives expression (16),

$$
\begin{aligned}
& B(v) \cdot \frac{1}{B(v)}=\sqrt{\frac{c-v}{c+v}} \cdot \sqrt{\frac{c+v}{c-v}}=1, \\
& \ln \left(\sqrt{\frac{c-v}{c+v}} \cdot \sqrt{\frac{c+v}{c-v}}\right)=\ln (1) .
\end{aligned}
$$

Thus,

$$
\begin{aligned}
& \ln \left(\sqrt{\left.\frac{c-v}{c+v}\right)}+\ln \left(\sqrt{\left.\frac{c+v}{c-v}\right)}\right.\right. \\
& =2 n \pi \boldsymbol{i}, \\
& =2 n \pi \cdot \frac{\psi(x, t)-\psi^{*}(x, t)}{2 A \sin (x, t)}, \\
& =2 n \pi \cdot\left(\frac{x p-p x}{h / 2 \pi}\right), \\
& =2 n \pi \cdot \lim _{\boldsymbol{v} \rightarrow \infty} \sqrt{\frac{c \pm v}{c \mp}}, \\
& =2 n \pi \cdot \sqrt{\frac{m^{2} c^{4}}{p^{2} c^{2}-E^{2}}},
\end{aligned}
$$

$$
\begin{aligned}
& =2 n \pi \cdot \sqrt{\frac{(c t)^{2}}{s^{2}-\left(x^{2}+y^{2}+z^{2}\right)}}, \\
& =2 n \pi \cdot \sqrt{\frac{\Lambda g_{u v}}{G_{\mu v}-k T_{u v}}},
\end{aligned}
$$

where $n=$ integers, $\pi=$ circular constant, $\boldsymbol{i}=$ imaginary unit, $x=$ position, $p=$ momentum, $\psi(x, t)=$ wave function, $\psi^{*}(x, t)=$ conjugate complex of $\psi(x, t), \sin (x, t)=$ sine function, $A=$ amplitude, $g_{\mu v}=$ metric tensor, $G_{\mu v}=$ Einstein tensor, $T_{u v}=$ energy-momentum tensor, $\kappa=$ Einstein's constant of gravitation, $\Lambda=$ cosmological constant, double-sign corresponds.

Expressions (17) (19) seem to suggest a hypothetical relationship between the relativistic expansion of the toy space model and the quantum phenomenon. Expressions (17) (23) seem to suggest a hypothesis that the toy space model using Bondi $\mathrm{K}$-factor relates some natural phenomena through the imaginary unit.

The imaginary unit is given by the mathematical application of the infinite velocity to Bondi $\mathrm{K}$-factor (20). The velocity is defined by dividing distance traveled by time elapsed. Since it is impossible to define the infinite distance, the infinite velocity is hypothetically and forcedly defined by dividing the given distance traveled by no time elapsed. Thus, the infinite velocity seems to be hypothetically interpreted as the simultaneous existence of different states (superposition), which seems to be something like oneness, wholeness, or ubiquity. However, the infinite velocity breaks the Lorentz invariance.

John Bell answered in the interview with him (Davies and Brown, 1986), "Behind the apparent Lorentz invariance of the phenomena, there is a deeper level which is not Lorentz invariant. …. The reason I want to go back to the idea of an aether here is because in these EPR experiments there is the suggestion that behind the scenes something is going faster than light. …. It is as if there is some kind of conspiracy, that something is going on behind the scenes which is not allowed to appear on the scenes. ..... And that's very ironic, because it is precisely his own theory of relativity which creates difficulties for this interpretation of the quantum theory (which is in the spirit of Einstein's unconventional view of quantum mechanics)." Alain Aspect answered in the interview with him (Davies and Brown, 1986), "However, if you mean that in some picture of the world that you want to construct, you can include some kind of faster than light mathematical object, then perhaps, yes, it could be a possibility. But you cannot use this mathematical construction for practical faster-than-light signalling. ..... However, all that they demolish is the possibility of having hidden variable theory based on Einstein's ideas such as separability."

Needless to say, the quantum phenomenon is considered basic to molecular biology in animal agriculture. Expressions (17) (23) seem to suggest a hypothesis that the quantum phenomena squared leads to the phe- 
nomena observed actually.

\section{Hypothetical phenomena in the birth point of the toy space model}

If substituting $c$ for $v$ in expression (8), then the birth point of the toy space model seems to be hypothetically given by expression (24),

$$
\begin{aligned}
B(c) \cdot \frac{1}{B(c)} & =\sqrt{\frac{c-c}{c+c}} \cdot \sqrt{\frac{c+c}{c-c}} \\
& =\sqrt{\frac{0}{2 c}} \cdot \sqrt{\frac{2 c}{0}} \\
& =0 \cdot \infty=1 .
\end{aligned}
$$

Expression (24) seems to suggest a massless state $(v=c)$ when the size of the toy space model is zero $[B(c)=0]$ at the birth point. Does the state of $\infty$ in (24) seem to suggest a hypothesis that the energy density is $\infty$ at the birth point of the toy space model? Does ' 1 ' in (24) seem to suggest a hypothesis that the singularity at the birth point is under the control of the invariant?

The natural logarithm of expression (24) gives expressions (25) (26) and (18) (19),

$$
\begin{aligned}
\ln (0 \cdot \infty) & =\ln (1), \\
-\infty+\infty & =2 n \pi \boldsymbol{i}, \\
& =2 n \pi \cdot \frac{\psi(x, t)-\psi^{*}(x, t)}{2 A \sin (x, t)}, \\
& =2 n \pi \cdot\left(\frac{x p-p x}{h / 2 \pi}\right) .
\end{aligned}
$$

Expressions (24) (26) and (18) (19) seem to suggest a hypothesis that the toy space model was born from the quantum phenomenon (or from the seeming zero) [(18 (19)] through something like a renormalization of infinities (26). However, this hypothetical phenomenon suggested by the toy space model is not consistent with preceding studies on the cosmic birth (Vilenkin, 1982; Hartle and Hawking, 1983).

\section{Conclusions}

This study shows that the toy space model using Bondi $\mathrm{K}$-factor has various problems when compared with the standard model of cosmic expansion.

\section{REFERENCES}

Albrecht, A. and P. J. Steinhardt 1982 Cosmology for grand unified theories with radiatively induced symmetry breaking. Phys. Rev. Lett., 48: 1220-1223

Barrow, J. D. 2011 The Book of Universes (The Bodley Head, London). Japanese Translation by H. Hayashi and M. Hayashi for publication (2013), Seidosha Inc., Tokyo.
Bedran, M. L. 2002 A comparison between the Doppler and cosmological redshifts. Am. J. Phys., 70: 406-408

Ben-Amots, N. 2011 Relativistic radial expansion: do we need dark energy? J. Phys.: Conf. Ser., 330: 1-11

Blackman, V. H. 1919 The compound interest law and plant growth. Ann. Bot., 33: 353-360

Bondi, H. 1964 Relativity and Common Sense (Doubleday and Company Inc., USA). Japanese Translation by T. Yamanouchi for publication (1967), Kawade Shobo Publishers, Tokyo.

Brody, S. 1945 Time relations of growth of individuals and populations. In "Bioenergetics and Growth - with special reference to the efficiency complex in domestic animals", Reinhold Publishing Corporation, New York, pp. 484-574

Bunn E. F. and D. W. Hogg 2009 The kinematic origin of the cosmological redshift. arXiv:0808.1081v2

Chodorowski, M. J. 2006 Is space really expanding? A counterexample. arXiv.astro-ph/0601171v2

Choi, H. 2010 Transition of expansion acceleration of the universe through negative mass. viXra:1008.0045

Davies, P. C. W. and J. R. Brown 1986 The Ghost in the Atom. Cambridge University Press, Cambridge.

Davis, T. M. 2010 Is the universe leaking energy? Sci. Am., 7: 33-39

Farley, F. J. M. 2010 Does gravity operate between galaxies? Observational evidence re-examined. Proc. R. Soc. A, 466 3089-3096

Francis, M. J., L. A. Barnes, J. B. James and G. F. Lewis 2007 Expanding space: the root of all evil? arXiv:0707.0380v1

Guth, A. H. 1981 Inflationary universe: a possible solution to the horizon and flatness problems. Phys. Rev. D, 23: 347-356

Hartle, J. B. and S. W. Hawking 1983 Wave function of the universe. Phys. Rev. D, 28: 2960-2975

Kaiser, N. 2013 Astronomical redshifts and the expansion of space. arXiv:1312.1190v1

Kazanas, D. 1980 Dynamics of the universe and spontaneous symmetry breaking. Astrophys. J., 241: L59-L63

Kipreos, E. T. 2014 Implications of an absolute simultaneity theory for cosmology and universe acceleration. PLOS ONE, 9: 1-20

Komatsu, E., J. Dunkley, M. R. Nolta, C. L. Bennett, B. Gold, G. Hinshaw, N. Jarosik, D. Larson, M. Limon, L. Page, D. N Spergel, M. Halpern, R. S. Hill, A. Kogut, S. S. Meyer, G. S. Tucker, J. L. Weiland, E. Wollack, and E. L. Wright 2009 Five-year Wilkinson microwave anisotropy probe observations: cosmological interpretation. Astrophys. J. Suppl. Ser. 180: $330-376$

Konushko, V. 2014 The mystery of the universe: Accelerated expansion, dark energy and dark matter. Int. J. High Energy Phys., 1: $38-48$

Lavenda, B. H. 2000 Special relativity via modified Bessel functions. Z. Naturforsch., 55a: 745-753

Li, M. 2014 Understanding the gravitational and cosmological redshifts as Doppler shifts by gravitational phase factors. arXiv: $1401.5337 \mathrm{v} 2$

Linde, A. D. 1982 A new inflationary universe scenario: a possible solution of the horizon, flatness, homogeneity, isotropy and primordial monopole problems. Phys. Lett., 108B: 389-393

MacCallum, M. A. H. 2015 Milestones of general relativity: Hubble's law (1929) and the expansion of the universe. arXiv:1504. 03606v1

Macleod, A. 2004 Redshift and energy conservation. arXiv.org/ pdf/physics/0407077

Matsubara, T. 2013 Cosmology. In "Encyclopedia of Astronomy (Blue Backs B1806)", ed. by Y. Taniguchi, Kodansha, Ltd., Tokyo (written in Japanese)

May, X. and P. Yu 2013 Cosmology should directly use the Doppler's formula to calculate the red shift of Ia supernova. Int. J. Astron. Astrophys., 3: 303-317

Peacock, J. A. 2008 A diatribe on expanding space. arXiv:0809. $4573 \mathrm{v} 1$

Perlmutter, S. 2011 Measuring the acceleration of the cosmic expansion using supernovae. Nobel Lecture (December 8 , 2011), pp. 3-40 
Perlmutter, S., G. Aldering, G. Goldhaber, R. A. Knop, P. Nugent, P. G. Castro, S. Deustua, S. Fabbro, A. Goobar, D. E. Groom, I. M. Hook, A. G. Kim, M. Y. Kim, J. C. Lee, N. J. Nunes, R. Pain, C. R. Pennypacker, R. Quimby, C. Lidman, R. S. Ellis, M. Irwin, R. G. McMahon, P. Ruiz-Lapuente, N. Walton, B. Schaefer, B. J. Boyle, A. V. Filippenko, T. Matheson, A. S. Fruchter, N. Panagia, H. J. M. Newberg and W. J. Couch 1999 Measurements of $\Omega$ and $\Lambda$ from 42 high-redshift supernovae. Astrophys. J., 517: $565-586$

Pierseaux, Y. 2014 Minkowskian solution of general relativity with cosmological constant and the accelerating universe. J. Mod. Phys., 5: 1725-1732

Rabounski, D. 2009 Hubble redshift due to the global nonholonomity of space. The Abraham Zelmanov J., 2: 11-28

Rebhan, E. 2012 Cosmic inflation and big bang interpreted as explosions. arXiv:1211.1006v1

Riess, A. G., A. V. Filippenko, P. Challis, A. Clocchiatti, A. Diercks, P. M. Garnavich, R. L. Gilliland, C. J. Hogan, S. Jha, R. P. Kirshner, B. Leibundgut, M. M. Phillips, D. Reiss, B. P. Schmidt, R. A. Schommer, R. C. Smith, J. Spyromilio, C. Stubbs, N. B. Suntzeff and J. Tonry 1998 Observational evidence from supernovae for an accelerating universe and a cosmological constant. Astron. J., 116: 1009-1038

Santilli, R. M. 2015 Representation of galactic dynamics via iso- shifts without universe expansion, dark matter and dark energy Am. J. Mod. Phys., 4: 26-43

Sato, K. 1981 First-order phase transition of a vacuum and the expansion of the universe. Mon. Not. R. Astron. Soc., 195 467-479

Shimojo, M. 2014 An application of Bondi K-factor to the preliminary investigation into some natural phenomena. J. Fac. Agr. Kyushu Univ., 59: 301-303

Shimojo, M. 2015 A size expansion analysis using a toy model based on the equivalence between Bondi $\mathrm{K}$-factor and exponential function. J. Fac. Agr., Kyushu Univ., 60: 93-96

Sitnikov, L. S. 2006 Hubble's law and superluminity recession velocities. arXiv.astro-ph/0602102v2

Starobinsky, A. A. 1980 A new type of isotropic cosmological models without singularity. Phys. Lett., 91B: 99-102

Tegmark, M. 2014 Our Mathematical Universe. Alfred A. Knopf, Inc., New York.

Vilenkin, A. 1982 Creation of universes from nothing. Phys. Lett., 117B: $25-28$

Wagh, S. M. 2013 Doppler's effct, gravity and cosmology. J. Mod. Phys., 4: 102-104

Weinberg, S. 2008 Cosmology. Oxford University Press Inc., New York. 